\title{
MATHEMATICAL MODELLING OF VARICELLA SPREAD IN SLOVAKIA
}

\author{
Jana Zibolenová1, Veronika Szabóová1, Tibor Baška ${ }^{1}$, Daniel Ševčovič², Henrieta Hudečková1 \\ 'Department of Public Health, Jessenius Faculty of Medicine in Martin, Comenius University in Bratislava, Martin, Slovak Republic \\ 2Department of Applied Mathematics and Statistics, Faculty of Mathematics, Physics and Informatics, Comenius University in Bratislava, Bratis- \\ lava, Slovak Republic
}

\section{SUMMARY}

Aim: Varicella is a highly contagious disease. In Slovakia, 15,000-30,000 cases are reported annually. However, vaccination against varicella is not widely used in Slovakia. The aim of this paper is to predict the influence of vaccination administrated in different ages on the disease occurrence in different age groups.

Methods: Deterministic, age-stratified SIR (susceptible-infected-recovered) model with stable population was used for prediction of varicella spread in Slovakia.

Results: Vaccination of a group designated as 0 influences the proportion of susceptible and infected persons in all age groups, vaccination of 10 years old ones affects only the proportion of susceptible and infected individuals older than 10 years.

Conclusion: The increase of vaccination coverage should have a positive impact on the incidence of varicella in Slovakia. In case of vaccination coverage of 0 group in the range of $30-75 \%$, it is necessary to pay attention to the protection of older children and adults.

Key words: varicella, vaccination, mathematical modelling, Slovakia, deterministic model

Address for correspondence: J. Zibolenová, Department of Public Health, Jessenius Faculty of Medicine in Martin, Sklabinská 26, 03601 Martin, Slovak Republic. E-mail: jana.zibolenova@gmail.com

\section{INTRODUCTION}

Varicella (chickenpox) as a highly contagious disease of children is caused by varicella zoster virus. The risk of complications depends on age and immune status. Source of infection is a person with manifest or inapparent infection. The disease is transmitted from person to person by direct contact and droplets $(1,2)$. Incubation period ranks from 14 to 21 days. The virus is highly contagious and can be spread 1-2 days before the rash appears until all blisters form crusts (usually 3-7 days) (3). Following chickenpox infection, the virus may remain latent in neural ganglia and after reactivation can be manifested as herpes zoster disease (shingles).

In Slovakia, 15,000-35,000 cases are reported annually $(4,5)$. Vaccination is the most effective method of prevention against varicella. Two types of varicella vaccine are currently available in the Slovak Republic. Tetravalent vaccine against measles, mumps, rubella and varicella (MMRV) can be provided either for children 15-18 months old, or in 11 th year within mandatory regular vaccination. The monovalent vaccine can be used for vaccination of other people including adult persons. Vaccine is administered in two-dose schedule. In some countries (e.g. US, Germany), unlike Slovakia, it is included into mass vaccination. Vaccination against varicella has been available in the Slovak Republic only for a short time and only a small part of the population has been vaccinated. It is expected that significantly increased vaccination coverage could potentially cause decline in morbidity rate of the disease. The aim of this study is to predict the impact of vaccination on the occurrence of disease in different age groups using methods of mathematical epidemiology.

\section{MATERIALS AND METHODS}

\section{Population Structure}

Deterministic, age-stratified SIR (susceptible-infected-recovered) model (Fig. 1) was used for description of circulation of varicella in the Slovak Republic (6). Population with constant size of 5,000,000 inhabitants was due to comparability with data from EPIS (Epidemiological Information System) (5) divided into three child age groups ( $0-4$ years, 5-9 years, 10-14 years), each of 300,000 children, and the rest of 4,100,000 inhabitants were considered adults (15+ years). The population was further divided into three groups according to the infectious status:

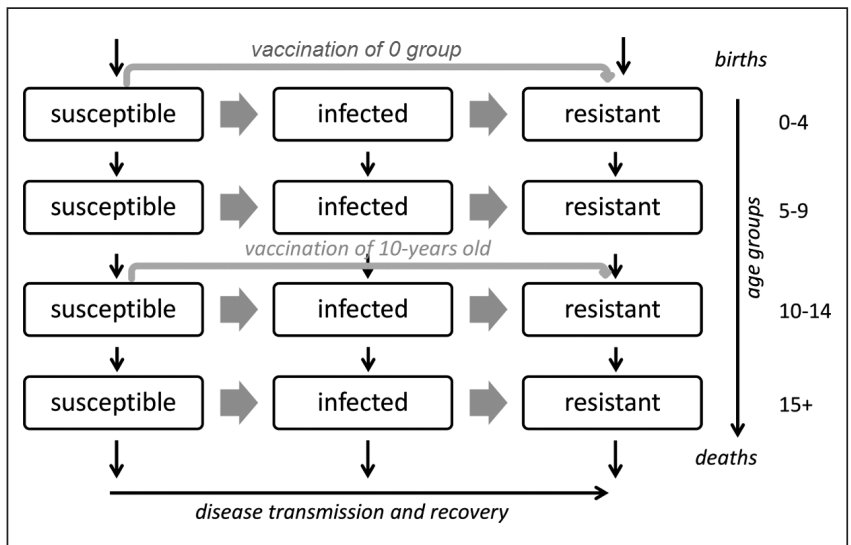

Fig. 1. Structure of SIR (susceptible-infected-removed) model used for prediction of varicella spread in Slovakia. 
$\mathrm{S}$ - susceptible, I - infected, R - resistant (sometimes called recovered). As susceptible is considered an individual who has not yet been infected and is at risk of infection; infected is an individual who can transmit pathogen to another individual; and resistant individual is a person, who has developed immunity after administration of vaccine or after overcoming the disease and is no longer susceptible to infection.

\section{Transitions across the Groups}

There are several types of transition across the groups in the model: ageing, natality, mortality, disease transmission, recovery, and vaccination. Mortality and birth rates have continuous character with the same value of $12 \%$ annually (i.e. 60,000 births and deaths annually). The influx of new susceptible individuals was provided by births and the size of population was constant due to deaths (only in adult group). Every year (on the 365th day) one fifth of children (60,000 children) from every child age group passes to the next age group. This transition copies at least partially the mechanism of transfer of pupils and students across classes and schools.

Transitions due to disease transmission were described by transmission parameters, which are characterized by WAIFW matrix - Who Acquires Infection from Whom $(6,7)$. Recovery rate is an inverse proportion of duration of infectious period.

Vaccination of a group designated as 0 was provided continuously together with exchange of population - part of individuals entering the population remained susceptible, the other part has become immune. Vaccination of 10 year olds was different - it was performed once a year together with transition of individuals from 5-9 years group to 10-14 years group.

The following set of equations was used for description of propagation of disease.

$$
\begin{aligned}
& \frac{d S_{a}}{d t}=\pi_{a}\left(1-p_{1}\right)-S_{a} \sum_{i=1}^{4} \beta_{i a} I_{i}-\frac{\mu_{a} S_{a}}{S_{a}+I_{a}+R_{a}} \\
& \frac{d I_{a}}{d t}=S_{a} \sum_{i=1}^{4} \beta_{i a} I_{i}-\gamma I_{a}-\frac{\mu_{a} I_{a}}{S_{a}+I_{a}+R_{a}} \\
& \frac{d R_{a}}{d t}=\pi_{a} p_{1}+\gamma I_{a}-\frac{\mu_{a} R_{a}}{S_{a}+I_{a}+R_{a}} \\
& a \in\{0-4, \quad 5-9, \quad 10-14, \quad 15+\}
\end{aligned}
$$

$S_{a}$ is a proportion of susceptible individuals in age group $a, I_{a}$ is a proportion of infected, $R_{a}$ is a proportion of immune individuals of total population, $t$ is a time. $\pi_{a}$ is a birth rate, which has nonzero value only for the first age group, by contrast $\mu_{a}$ (mortality rate) has zero value for all age groups except $15+$ years old. $\mathrm{p}_{1}$ is a proportion of vaccinated individuals from 0 group (in fact, the proportion of those with developed immunity after administration of vaccine), $\beta_{i a}$ are transmission parameters between age groups $a$ and $i$ (WAIFW matrix) and $\gamma$ is a recovery rate (1/length of infectious period). Similar model has been already published (6).

Every year (on the 365th day) one fifth of children from each children age group passes to the next age group, with preserving infectious status (not considering vaccination of 10 years old)

$$
\begin{aligned}
& Q_{0-4}:=Q_{0-4}-\frac{1}{5} Q_{0-4} \\
& Q_{5-9}:=\frac{1}{5} Q_{0-4}+Q_{5-9}-\frac{1}{5} Q_{5-9} \\
& Q_{10-14}:=\frac{1}{5} Q_{5-9}+Q_{10-14}-\frac{1}{5} Q_{10-14} \\
& Q_{15+}:=\frac{1}{5} Q_{10-14}+Q_{15+} \\
& Q \in\{S, I, R\}
\end{aligned}
$$

In case of considering vaccination of 10 years old children, equations for susceptible and immune children in the age group of 10-14 years are changed

$$
\begin{aligned}
& S_{10-14}=\frac{1}{5} S_{5-9}-\frac{1}{5} p_{2} S_{5-9}+S_{10-14}-\frac{1}{5} S_{10-14} \\
& R_{10-14}=\frac{1}{5} R_{5-9}+\frac{1}{5} p_{2} S_{5-9}+R_{10-14}-\frac{1}{5} R_{10-14}
\end{aligned}
$$

$p_{2}$ is vaccination coverage of 10 years old children.

WAIFW (Who Acquires Infection From Whom) matrix reflects the structure of effective contacts in population among age groups. Diagonal elements reflect probability of acquiring infection from individual from the same age group, and other elements represent effective contacts across different age groups.

The structure of WAIFW matrix is symmetric

$$
\beta_{i a}=\left(\begin{array}{cccc}
k_{1} & k k_{2} & k_{3} & k_{4} \\
k k_{2} & k_{2} & k_{3} & k_{4} \\
k_{3} & k_{3} & k_{3} & k_{4} \\
k_{4} & k_{4} & k_{4} & k_{4}
\end{array}\right) .
$$

$k$ is a factor of sensitivity analysis and represent the coefficient of relative difference between transmission parameters $\beta_{12}$ and $\beta_{22}$. The values from 0.7 to 1.1 correspond with the assumption of nearly equal or lower number of effective contacts between the first and second age group than inside the second age group.

Subsequently, from the equation for calculation of age-specific force of infection $\lambda_{a}$ transmission parameters $\beta_{i a}$ were calculated

$$
\lambda_{a}=\sum_{i=1}^{4} \beta_{i a} \tilde{I}_{i}
$$

where $\tilde{I}_{i}$ is an average proportion of infected individuals in the $i^{\text {th }}$ age group in the state of endemic equilibrium (7).

\section{Input Parameters}

Demographic input parameters (natality, mortality rate, size of age groups) were selected to correspond with demographic situation in Slovakia (Table 1). The age-specific forces of infection (the rate at which susceptible individuals become infected per unit time) for child age groups are obtained from sero-epidemiology study (8). For the 4th age group (15+ years old) exact data on force of infection are not available from Slovakia, so we estimated this value using foreign data $(9,10)$. In the model equations the values of force of infection were not directly used, but the transmission 
Tab. 1. Input parameters used in the modelling of effect of vaccination against varicella in Slovakia

\begin{tabular}{|c|c|c|}
\hline \multirow[t]{5}{*}{ Population size by age groups } & Total & $5,000,000$ inhabitans \\
\hline & $0-4$ years & 300,000 inhabitans \\
\hline & $5-9$ years & 300,000 inhabitans \\
\hline & $10-14$ years & 300,000 inhabitans \\
\hline & $15+$ years & $4,100,000$ inhabitans \\
\hline Natality $\left(\pi_{a}\right)-$ only for $0-4$ years age group & \multicolumn{2}{|l|}{$12 \%$ of total population per year } \\
\hline Mortality $\left(\mu_{\mathrm{a}}\right)-$ only for $15+$ years age group & \multicolumn{2}{|l|}{$12 \%$ of total population per year } \\
\hline Transition between age groups (once a year) & \multicolumn{2}{|l|}{60,000 inhabitans per year } \\
\hline Life expectancy & \multicolumn{2}{|l|}{75 years } \\
\hline Infectiouse period (1/Y) & \multicolumn{2}{|l|}{7 days (3) } \\
\hline \multirow{4}{*}{$\begin{array}{l}\text { The age-specific force of infection }\left(\lambda_{\mathrm{a}}\right) \text { characterizing the rate } \\
\text { at which susceptible individuals become infected per unit time } \\
\text { (in brackets are } 95 \% \text { confidence intervals for child age groups, } \\
\text { and minimum and maximum for } 15+\text { years age group) }\end{array}$} & $0-4$ years & $0.156(0.140-0.174)$ per year $(8)$ \\
\hline & $5-9$ years & $0.253(0.223-0.286)$ per year $(8)$ \\
\hline & $10-14$ years & $0.137(0.111-0.165)$ per year $(8)$ \\
\hline & $15+$ years & $0.013(0.008-0.018)$ per year (estimation) \\
\hline \multirow{4}{*}{$\begin{array}{l}\text { Transmission parameters }\left(k_{k}\right) \text { characterizing the structure of } \\
\text { effective contacts in population among age groups expressed } \\
\text { in number of effective contacts per day (in brackets minimum } \\
\text { and maximum). They are calculated from age-specific forces } \\
\text { of infection (7) }\end{array}$} & $\mathrm{k}_{1}$ (correspond to $\beta_{11}$ ) & $0.975(0.120-1.935)$ per day \\
\hline & $\mathrm{k}_{2}$ (correspond to $\beta_{22}, \beta_{12}, \beta_{21}$ ) & $2.741(2.430-4.147)$ per day \\
\hline & $\mathrm{k}_{3}$ (correspond to $\left.\beta_{13}, \beta_{23}, \beta_{33}, \beta_{32}, \beta_{31}\right)$ & $1.308(1.254-1.901)$ per day \\
\hline & $k_{4}$ (correspond to $\beta_{14}, \beta_{24}, \beta_{34}, \beta_{44}, \beta_{43}, \beta_{42}, \beta_{41}$ ) & $0.091(0.088-0.199)$ per day \\
\hline Factor in WAIFW matrix used for sensitivity analysis (k) & \multicolumn{2}{|l|}{$0.7-1.1$} \\
\hline $\begin{array}{l}\text { Vaccination coverge for } 0 \text { group and } 10 \text { years old individuals } \\
\left(p_{1}, p_{2}\right)\end{array}$ & \multicolumn{2}{|c|}{$0 \%, 15 \%, 30 \%, 45 \%, 60 \%, 75 \%, 90 \%$ of susceptible individuals from the given age group } \\
\hline
\end{tabular}

parameters used in equations were calculated from the force of infection (see above) (7).

Sensitivity analysis was performed according to the force of infection and partially through WAIFW matrix structure. Each age-specific force of infection has a relevant parameter expressed as a confidence interval (Table 1). Within its range, five limiting points equally distributed were chosen. By this way $5^{4}=625$ simulations were generated. Furthermore, using WAIFW structure, five extra simulations were added to each previously mentioned simulations. Therefore, there were $5^{5}=3,125$ simulations generated for each vaccination coverage.

The initial conditions corresponded with endemic state equilibrium. Simulations were generated using Matlab software (11).

\section{Time Horizon}

In time $\mathrm{t}=0$, the initial conditions for proportions of susceptible, infected and resistant groups were based on average proportion of susceptible, infected and resistant individuals with fixed age-specific forces of infection. To prevent high oscillation of proportions of susceptible, infected and resistant groups, the introduction of vaccination was set 30 years after proceeding the start of simulation. In this period, proportions of susceptible, infected and resistant individuals were approaching the endemic equilibrium. Length of prediction was 50 years from the beginning of vaccination. In terms of transients, we examined the proportions of susceptible, infectious and immune individuals within 10 years from the start of the vaccination (short-term) and between 11 to 50 years following the start of the vaccination (long-term).

\section{Output of the model}

The primary output of the model represented the course of the functions of proportions of susceptible, infected and resistant individuals depending on time. However, this output cannot be directly compared to the reality. Therefore, the average annual number of infected and average proportion of susceptible individuals in the short-term ( $0-10$ years since introduction of vaccination) and long-term horizon (11-50 years following the start of the vaccination) for each simulation was calculated. Results for the infected were also adjusted for length of infectious period. Results displayed in Fig. 2a, b and Fig. 3a, b are average values (minimum and maximum error bars) through all simulations at the given vaccination coverage.

\section{RESULTS}

\section{Vaccination of 0 Group}

In the first modelled strategy, only the vaccination of 0 group was considered. In the long-term and also in the short-term period the proportion of susceptible individuals in $0-4$ age group is decreasing with increasing vaccination level. In other age groups, at the beginning there is an increase in proportion of susceptible individuals and reaching the level of vaccination coverage approximately $60 \%$ is followed by a decrease in proportion of the susceptible (Fig. 2a). In the short-term aspect these changes are not so pronounced compared to the long-term.

The increase in vaccination coverage of 0 group leads to decrease in incidence in all age groups in the long-term (Fig. 3a) as well as short-term period. When the vaccination coverage is 
around $50 \%$, the lower incidence of the disease is associated with increased prevalence of susceptible individuals because of lower natural circulation of virus in the population (Fig. 4a, b). When the vaccination coverage is above $75 \%$, the disease occurs in the long-term horizon only sporadically. However, relative high proportion of susceptible persons still remains in the population. These are protected by herd immunity.

The increase in vaccination coverage also leads to increase in mean age of infected individuals in the long-term horizon. The reason is the most intensive decrease in number of infections in the lowest age group. In other age groups, there is also a decrease in number of infections, although less significant compared to the lowest age group. The shift of mean age of infected individuals is not associated with an increase of average number of disease cases in adults.

\section{Vaccination of 10 Years Old Children}

In the second modelled strategy, only the vaccination of 10 years old children was considered. This vaccination was performed once a year along with transition of individuals from 5-9 years age group to 10-14 years age group. Vaccination of 10 years old children does not substantially affect the proportion of susceptible individuals and the incidence of the disease in two younger age groups. The increase of vaccination coverage in 10 years old children leads to a decrease in proportion of susceptible individuals and number of cases of varicella in 10-14 and 15+

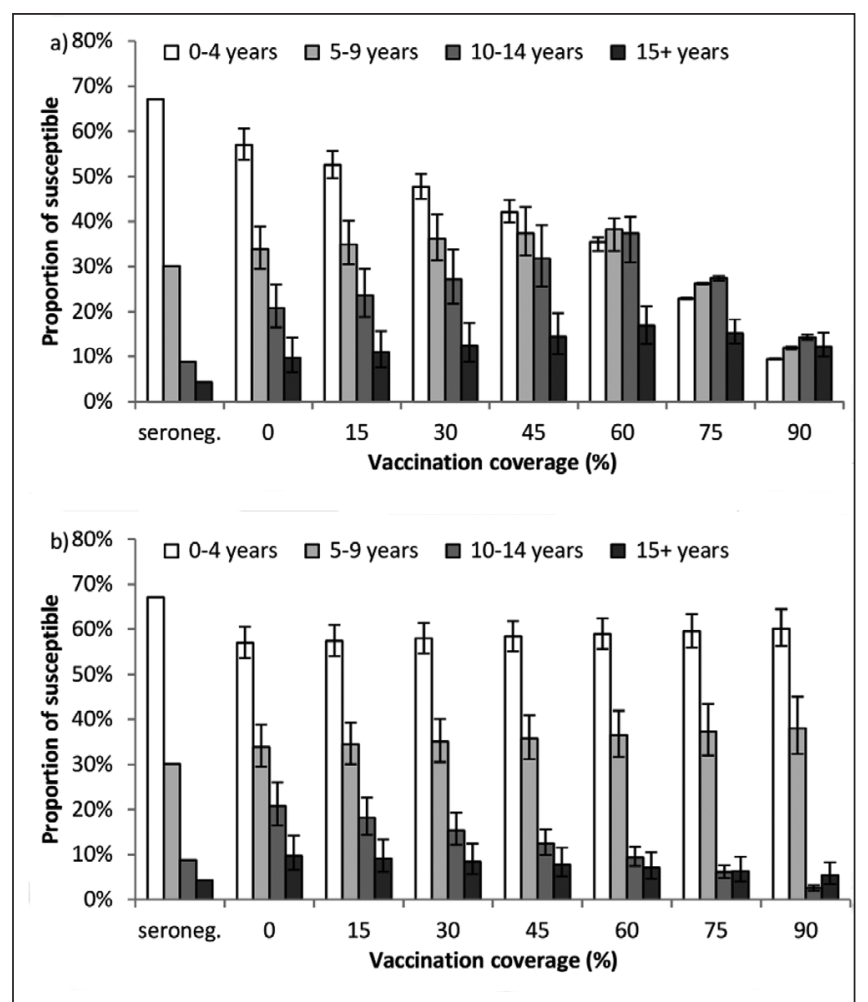

Fig. 2a, b. Average proportions of susceptible individuals in appropriate age groups in the period 10-50 years after introduction of varicella vaccination for 0 group (a) and 10 years old children (b). In the first set of bars there is a proportion of seronegative in appropriate age groups based on seroepidemiology study (8). Error bars correspond to range (minimum and maximum) obtained from sensitivity analysis. years age groups in the long-term aspect (Fig. 2b). In the shortterm aspect the group of $15+$ years old is not significantly affected and the changes in 10-14 years age group are not so profound as in the long-term aspect. The decrease in incidence is not as dramatic as in the first strategy, because adults can be infected from children (Fig. 3b). The mean age of infected individuals is not significantly influenced, rather a slight decline in the longterm aspect can be seen.

\section{DISCUSSION}

The mathematical modelling of spread of infectious diseases has generally its advantages and limitations. Many authors have dealt with modelling of varicella. We can mention for example model from Halloran et al. (12). It was published shortly before the introduction of mass vaccination against varicella in the US. Authors analyzed the influence of widespread vaccination in population on age-specific incidence of varicella. According to their results, although mass vaccination could possibly lead to shift of disease cases into higher age groups, the overall effect of vaccination should be considered positive. Of course, the number and age distribution of cases depend on characteristics of vaccine and other parameters.

A similar, but slightly more complicated model was used in analysis carried out by Brisson et al. (9). This model was used to predict the impact of different vaccination strategies on

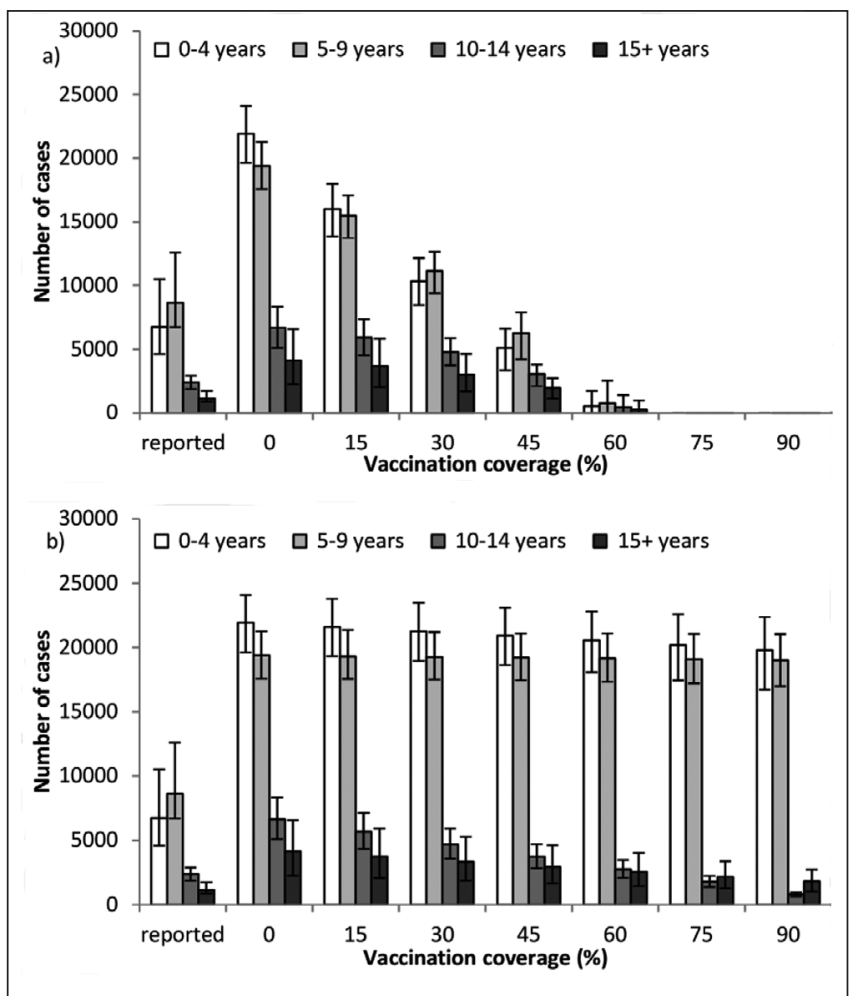

Fig. 3a, b. Average annual numbers of infected in each age group in the period 10-50 years after introduction of varicella vaccination for 0 group (a) and 10 years old children (b). In the first set of bars there are average (minimum and maximum) annual numbers of reported cases in Slovakia in period 1997-2012 (5). Error bars correspond to range (minimum and maximum) obtained from sensitivity analysis. 

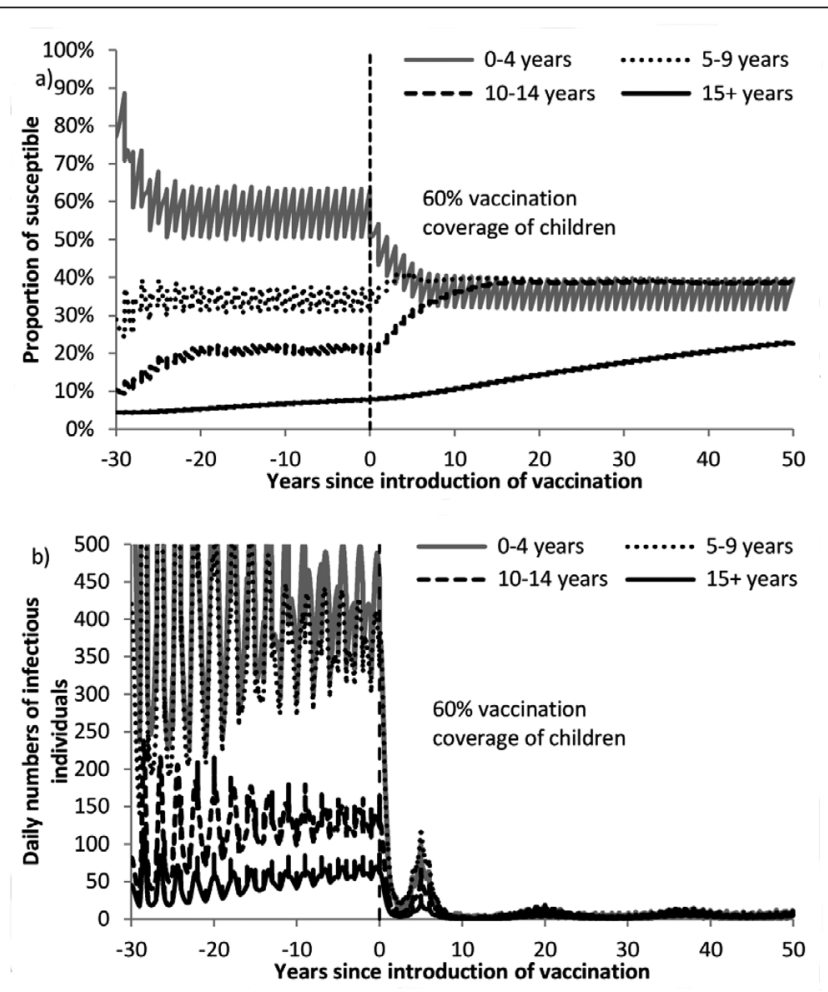

Fig. $\mathbf{4 a}, \boldsymbol{b}$. Development of proportion of susceptible (a) and daily numbers of infectious (not corrected for the length of infectious period) (b) in appropriate age groups; value of vaccination coverage in 0 group is $60 \%$, in 10 years old $0 \%$.

epidemiology of varicella and herpes zoster in Canada (9) and Australia (13). According to their work, the overall incidence and morbidity of varicella would be considerably reduced by mass vaccination of 12 months old children. Furthermore, adding a catch-up campaign in the first year for 1-11 year olds seems to be the most effective strategy to reduce varicella incidence and morbidity (both in the short and long term aspect). On the other hand, lowered occurrence of the disease could lead to weakened natural booster effect, resulting in transient increase of herpes zoster occurrence. Therefore, the overall effect of vaccination could be questionable $(9,14-16)$.

The real impact of vaccination on epidemiology of varicella has been observed in the US. In 1995, mass varicella vaccination was introduced and since that time a significant decline in morbidity and hospitalization rate in all age groups has been observed (17). The median age of infected individuals increased (18). However, one dose regime has not shown satisfactory efficiency, so two-dose vaccination schedule is therefore recommended.

Regarding the impact of varicella vaccination on herpes zoster disease (shingles), findings from the US are not unequivocal. Some of the studies confirmed increase in the incidence of the disease, some of the studies did not. Interestingly, the increase in the incidence of the shingles was also seen in countries where the mass vaccination against varicella had not been implemented. This suggests that a possible increase in incidence of the disease may be caused by other factors $(19,20)$.

The model used in this work is very simplified, but provides results consistent with results from previous published models and with actual situation in the countries with mass varicella vaccination. The model results with zero vaccination coverage can be compared with current varicella incidence in Slovakia $(4,5)$ and with the seroprevalence study $(8)$. The proportions of susceptible individuals in two younger age groups are similar to the proportion of seronegative individuals from the seroprevalence study. The proportion of susceptible individuals in two older age groups is higher in the model than in the seroprevalence study. Annual numbers of infected individuals (on average 52,053 cases per year) in the model are significantly higher than the number of actually reported cases (in average 18,890 cases per year in the period from 1997-2012) (21). This difference can be explained by underreporting, so the modelled numbers of cases are probably closer to reality than reported cases. In Slovakia, in the last 15 years, the number of births was between 50,000-60,000 per year. With the assumption that almost everybody had suffered from the disease before adulthood, the annual number of cases should approach the value of birth rate.

This simplified model assumes hundred percent protection rate following vaccination and lifelong immunity (after vaccination and after overcoming the disease). But this assumption is not real since not in all vaccinated individuals effective immune response is gained and part of them remains susceptible. To appropriately interpret our results, it is important to emphasize, that the modelled vaccination protection does not correspond with the real situation. It does not represent real vaccination coverage but it refers only to those with developed effective immune response. Therefore, it is necessary to reach higher vaccination coverage than in the model situation to obtain the given protection rate.

Other source of potential bias in the model is an assumption of quasi-homogeneous population. Although the model at least partially takes into consideration the different level of mixing between and within age groups (WAIFW matrix), these age groups are not homogeneous and the probability of meeting two arbitrary individuals is not equal. It is not possible to deal effectively with this factor using deterministic models. However, such type of model provides acceptable results despite the above mentioned limitations.

Other source of inaccuracy in the model is an assumption that children are vaccinated when entering the population. The model could be more accurate by dividing group of $0-4$ years old into two (or more) age groups. But values of age-specific force of infection for these groups are not known. Moreover, maternal immunity should be considered, which lasts on average $2-4$ months after birth (22). During the period between the loss of maternal immunity and formation of a post-vaccination immunity, a child is susceptible to disease. But modification of the model by this way would considerably increase a complexity of the model and reduced its transparency.

Our findings are consistent with current recommendations of the official health authorities. The increase of vaccination coverage of children falling into 0 group should have a positive impact on the incidence of varicella in Slovakia in general, however, the introduction of mass vaccination against varicella is so complex issue that it is beyond the scope of this article to assess benefits from different points of view (economic, adverse effects etc.).

If the mass vaccination against varicella in Slovakia will be introduced, based on our findings, we could recommend to administer the first dose as soon as possible to avoid potential spread of the disease. If the current system of varicella vaccination scheme would remain unchanged, it is necessary to monitor 
the vaccination coverage to timely respond to any unfavourable changes which might especially endanger susceptible adults. The vaccination of older children does not influence infection rate in younger ones, however, it can at least partially prevent complications associated with the disease in higher age groups.

\section{Acknowledgements}

This work was supported by the Slovak Research and Development Agency under the contract No.APVV-0096-12 and by grant UK/69/2013.

\section{REFERENCES}

1. Hudečková H, Švihrová V. Vaccination. Martin: Osveta; 2013. (In Slovak.)

2. Hudečková H. Practical vaccinology not only for medical students. Žilina: Lajka; 2009. (In Slovak.)

3. Dražan D. Varicella. Pediatr Praxi. 2007;8(6):374-8. (In Czech.)

4. Annual report of the activities of the regional public health authorities in the Slovak Republic [Internet]. Bratislava: Public Health Authority of the Slovak Republic; 2012 [cited 2015 Apr 25]. Available from: http://www. uvzsr.sk/index.php?option $=$ com_content\&view $=$ category\&layout $=$ blog \&id $=25 \&$ Itemid $=34$. (In Slovak.)

5. Epidemiological Information System (EPIS) [Internet]. Bratislava: Public Health Authority of the Slovak Republic; 2015 [cited 2015 Apr 25]. Available from: http://www.epis.sk/?lang=sk-SK. (In Slovak.)

6. Keeling MJ, Rohani P. Modelling infectious diseases in humans and animals. Princeton: Princeton University Press; 2008.

7. Vynnycky E, White R. An introduction to infectious disease modelling. New York: Oxford University Press; 2010.

8. Nardone A, de Ory F, Carton M, Cohen D, van Damme P, Davidkin I, et al. The comparative sero-epidemiology of varicela zoster virus in 11 countries in the European region. Vaccine. 2007 Nov 7;25(45):7866-72.

9. Brisson M, Edmunds WJ, Gay NJ, Law B, De Serres G. Modelling the impact of immunization on the epidemiology of varicella zoster virus. Epidemiol Infect. 2000 Dec;125(3):651-69.
10. Mossong J, Putz L, Schneider F. Seroprevalence and force of infection of varicella-zoster virus in Luxembourg. Epidemiol Infect. 2004 Dec;132(6):1121-7.

11. Matlab R2013a. Natick (MA): MathWorks; 2013.

12. Halloran EM, Cochi SL, Lieu TA, Wharton M, Fehrs L. Theoretical epidemiologic and morbidity effects of routine varicella immunization of preschool children in the United States. Am J Epidemiol. $1994 \mathrm{Jul}$ $15 ; 140(2): 81-104$.

13. Gidding HF, Brisson M, Macintyre CR, Burgess MA. Modelling the impact of vaccination on the epidemiology of varicella zoster virus in Australia. Aust N Z J Public Health. 2005 Dec;29(6):544-51.

14. van Hoek AJ, Melegaro A, Zagheni E, Edmunds WJ, Gay N. Modelling the impact of a combined varicella and zoster vaccination programme on the epidemiology of varicella zoster virus in England. Vaccine. 2011 Mar 16;29(13):2411-20.

15. van Hoek AJ, Melegaro A, Gay N, Bilcke J, Edmunds WJ. The costeffectiveness of varicella and combined varicella and herpes zoster vaccination programmes in the United Kingdom. Vaccine. $2012 \mathrm{Feb}$ 1;30(6):1225-34.

16. Brisson M, Edmunds WJ. Varicella vaccination in England and Wales: cost-utility analysis. Arch Dis Child. 2003 Oct;88(10):862-9.

17. Schmid DS, Jumaan AO. Impact of varicella vaccine on varicella-zoster virus dynamics. Clin Microbiol Rev. 2010 Jan;23(1):202-17.

18. Guris D, Jumaan AO, Mascola L, Watson BM, Zhang JX, Chaves SS, et al. Changing varicella epidemiology in active surveillance sites - United States, 1995-2005. J Infect Dis. 2008 Mar 1;197 Suppl 2:S71-5.

19. Reynolds MA, Chaves SS, Harpaz R, Lopez AS, Seward JF. The impact of the varicella vaccination program on herpes zoster epidemiology in the United States: a review. J Infect Dis. 2008 Mar 1;197 Suppl 2:S224-7.

20. Leung J, Harpaz R, Molinari NA, Jumaan A, Zhou F. Herpes zoster incidence among insured persons in the United States, 1993-2006: evaluation of impact of varicella vaccination. Clin Infect Dis. $2011 \mathrm{Feb}$ 1;52(3):332-40.

21. Malobická E, Hudečková H. Varicela. Pediatria. 2014;9(6):303-5. (In Slovak.)

22. Leuridan E, Hens N, Hutse V, Aerts M, Van Damme P. Kinetics of maternal antibodies against rubella and varicella in infants. Vaccine. 2011 Mar 3;29(11):2222-6.

Received August 18, 2014 Accepted in revised form April 25, 2015 